\title{
$1 \quad$ Are protein-ligand complexes robust structures?
}

2 Maciej Majewski, ${ }^{\dagger}$ Sergio Ruiz-Carmona, ${ }^{\dagger}$ and Xavier Barril ${ }^{*}, \neq$

3 'Institut de Biomedicina de la Universitat de Barcelona (IBUB) and Facultat de Farmacia,

4 Universitat de Barcelona, Av. Joan XXIII 27-31, 08028 Barcelona, Spain

$5 \quad \ddagger$ Catalan Institution for Research and Advanced Studies (ICREA), Passeig Lluis Companys 23,

$6 \quad 08010$ Barcelona, Spain

7 Drug Design, Dynamic Undocking, Hydrogen Bond, Molecular Dynamics, Structural Stability. 


\section{ABSTRACT}

2 The predominant view in structure-based drug design is that small-molecule ligands, once bound

3 to their target structures, display a well-defined binding mode. While this is convenient from a

4 design perspective, it ignores the fact that structural stability (robustness) is not necessary for

5 thermodynamic stability (binding affinity). In fact, any potential benefit of a rigid binding mode

6 will have to be balanced against the entropic penalty that it entails. Surprisingly, little is known

7 about the causes, consequences and real degree of robustness of protein-ligand complexes. Here

8 we investigate two diverse sets of structures, comprising 79 drug-like and 27 fragment ligands,

9 respectively. We focus on hydrogen bond interactions (469 in total), as they have been described

10 as essential for structural stability. We find that $75 \%$ of complexes are anchored by at least one

11 robust hydrogen bond, the remaining $25 \%$ either form loose complexes or are constrained by other

12 interactions types. The first type of complexes generally combine a single anchoring point with

13 looser regions, thus balancing order and disorder. Completely constricted protein-ligand

14 complexes are rare and seem to fulfil a functional necessity. Structural stability analysis reveals a

15 hidden layer of complexity in protein-ligand complexes that should be considered in ligand design. 
Biomolecular systems present a large number of degrees of freedom and must find a suitable

3 balance between order and disorder. In the particular case of non-covalent complexes, they can

4 exist in a continuum spectrum of possibilities, ranging from the lock-and-key model to extreme

5 disorder. ${ }^{1,2}$ While the importance of target flexibility is well-appreciated in drug discovery, ${ }^{3}$ the

6 flexibility of small-molecule ligands in their bound state has attracted much less attention. Detailed

7 analyses reveal that ligands often retain residual mobility. ${ }^{4-6}$ However, changes in binding mode

8 are more the exception than the norm ${ }^{7,8}$ and ligand design based on rigid crystallographic

9 geometries has been remarkably successful. ${ }^{9}$ Perhaps for this reason, little is known about the

10 molecular mechanisms that control structural stability, to what extent do ligands preserve

11 flexibility or what are the energetic and functional consequences of rigidity.

12 It is important to note that structural stability (robustness) is fundamentally different from

13 thermodynamic stability (i.e. binding free energy; $\Delta G_{b i n d}$ ). This is eloquently exemplified in the

14 recent work by Borgia et al., where a protein-protein complex with picomolar affinity is shown to

15 lack structure. ${ }^{2}$ While $\Delta G$ bind has been the center of attention of scientific research for decades,

16 little attention has been paid to the factors that determine if a complex will be tight or loose. The

17 source of structural robustness must be sought on sharp (and possibly transitory) energetic barriers

18 that keep the atoms in their positions of equilibrium. Such hypothetical barriers, like the ones that

19 determine binding kinetics, could have their origin in intramolecular (i.e. conformational

20 rearrangement), bimolecular (e.g. repulsive transitional configurations) or many-body effects (e.g.

21 desolvation). ${ }^{10}$ But they will only provide structural stability if the barriers are steep and located

22 very close to the position of minimum energy. In that respect, hydrogen bonds (HBs) are ideal

23 candidates because they have strict distance and angular dependencies ${ }^{11}$ and are one of the most 
1 frequent interaction types in protein-ligand complexes. ${ }^{12}$ The contribution of HBs to $\Delta G_{\text {bind }}$ has

2 been largely debated in the literature. ${ }^{13-17}$ The current consensus is that it is highly variable and

3 context dependent, but their contribution to thermodynamic stability is $1.8 \mathrm{kcal}$ mol-1 at the most. ${ }^{14}$

4 However, due to desolvation, the transitional penalty of breaking a HB can be much larger. ${ }^{18}$

5 Indeed, we have shown that this is the case for water-shielded HBs, which can even act as kinetic

6 traps. ${ }^{19}$ More recently, we have also shown that formation of structurally robust intermolecular

7 HBs at specific positions is a necessary condition for binding, and have developed a method to

8 assess the robustness of individual HBs that is very effective in virtual screening applications. ${ }^{20}$

9 With this background, we decided to perform a systematic investigation of the possible role of

10 HBs as structural anchors of protein-ligand complexes. Our findings not only confirm a general

11 role of $\mathrm{HBs}$ as source of structural stability, but also offer a new perspective to understand and

12 design ligand-receptor complexes.

RESULTS AND DISCUSSION

15 Using Dynamic Undocking (DUck), an MD-based computational procedure, ${ }^{20}$ we have assessed

16 the robustness of every HB in a set of 79 drug-like protein-ligand complexes from the Iridium Data

17 Set. $^{21}$ Detailed information about the data set and the selection criteria is presented in

18 Supplementary Methods and Supplementary Table 1. Each HB was pulled to a distance of $5 \AA$,

19 according to the DUck protocol reported previously. ${ }^{20,22}$ In this way, we obtain a work value $\left(W_{Q B}\right)$

20 that reflects the cost of breaking each $\mathrm{HB}$. In other words, the $\mathrm{W}_{\mathrm{QB}}$ value indicates if the interaction

21 under investigation gives rise to a narrow (local) minimum in the free-energy landscape, and

22 estimates its depth. Based on our previous research, we define HBs as robust (i.e. capable of 
1 providing structural stability) if $W_{Q B}>6 \mathrm{kcal} \mathrm{mol}^{-1}$, labile if $W_{Q B}<4 \mathrm{kcal} \mathrm{mol}^{-1}$ and medium

2 otherwise.

3 The distribution of work values for the entire set of $345 \mathrm{HBs}$ ranges from 0 to $26 \mathrm{kcal} \mathrm{mol}^{-1}$,

4 with a of maximum probability in the 0-6 $\mathrm{kcal} \mathrm{mol}^{-1}$ region and a gradual decrease thereafter

5 (Fig.1a). Noteworthy, more than half HBs (57.4\%) are robust. In order to provide a critical

6 assessment of these results, we have sought correlation with experimental observables and have

7 also considered if $\mathrm{W}_{\mathrm{QB}}$ values might be dominated by the interaction energies. Larger $\mathrm{W}_{\mathrm{QB}}$ values

8 imply a narrower minimum and, thus, restricted mobility, which should translate into a more

9 localized electron density, that is, lower crystallographic B-factors. As B-factors are heavily

10 influenced by the refinement methods used and their absolute values can be meaningless, ${ }^{23,24}$ we

11 have normalized the B-factor of the ligand atom that makes the hydrogen bond relative to the

12 average B-factor of the whole ligand. Encouragingly, atoms forming HBs with larger $\mathrm{W}_{\mathrm{QB}}$ values

13 tend to have lower relative B-factors (Supplementary Fig.1). A second aspect to consider is

14 whether DUck calculations merely reflects short-range protein-ligand interaction, or - as intended

15 - it captures a global effect that considers enthalpic and entropic contributions from both the solute

16 and the solvent. Lack of correlation between interaction energies and $\mathrm{W}_{\mathrm{QB}}$ confirms that the latter

17 is true (Supplementary Fig.2). Of particular interest is to assess the effect of charge reinforcement

18 on $\mathrm{HBs}$, as the energetic, entropic and solvation terms of neutral hydrogen bonds and salt bridges

19 are drastically different. ${ }^{25}$ We have classified all HBs into neutral, mixed (ionic-neutral) and salt

20 bridges (Fig.2, Supplementary Table 2). We find that salt bridges are only very slightly skewed

21 towards more robust interactions than neutral HBs. The distributions were compared with two

22 sample Kolmogorov-Smirnov statistical test, yielding p-value of 0.08. Mixed types are completely

23 indistinguishable from neutral ones $(\mathrm{p}$-value $=0.42)$. Unexpectedly, the maximal values are equal 
1 across all three categories. Theoretically, ionic species could provide even larger energetic barriers

2 because their desolvation costs are much larger. We speculate that there may be no biological use

3 for them, as the maximal $W_{Q B}$ values observed here already ensure very robust and long-lived

4 structures.

5 The distribution of robust HBs is rather inhomogeneous across complexes, as they have 2.5 on

6 average, but a quarter of the complexes have none (Fig. 1b). Considering that structural stability is

7 not a requisite for tight binding and that HBs may not the only mechanism capable of providing

8 structural stability, it is striking that $75 \%$ of the complexes in this set are anchored through HBs.

9 A further $14 \%$ of complexes present medium values and only in 9 cases (11\%) all their HBs are

10 labile (Supplementary Fig.3). Two of those cases are very low affinity complexes. In the remaining

11 cases, structural stability might be provided by other mechanisms or may be lacking (see examples

12 in Supplementary Fig.3). It is important to note that the level of structural stability reported here

13 may be overestimated due to the composition of the data set, entirely derived from X-ray

14 crystallography, a technique that relies on order to solve structures. 


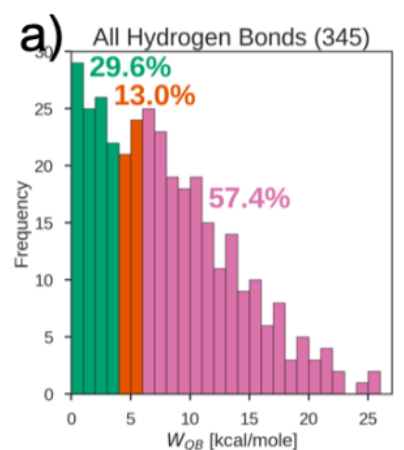

b) $\operatorname{Max} W_{Q B}$ in a complex (79)

C) Enzymes - all HBs (253)

d)
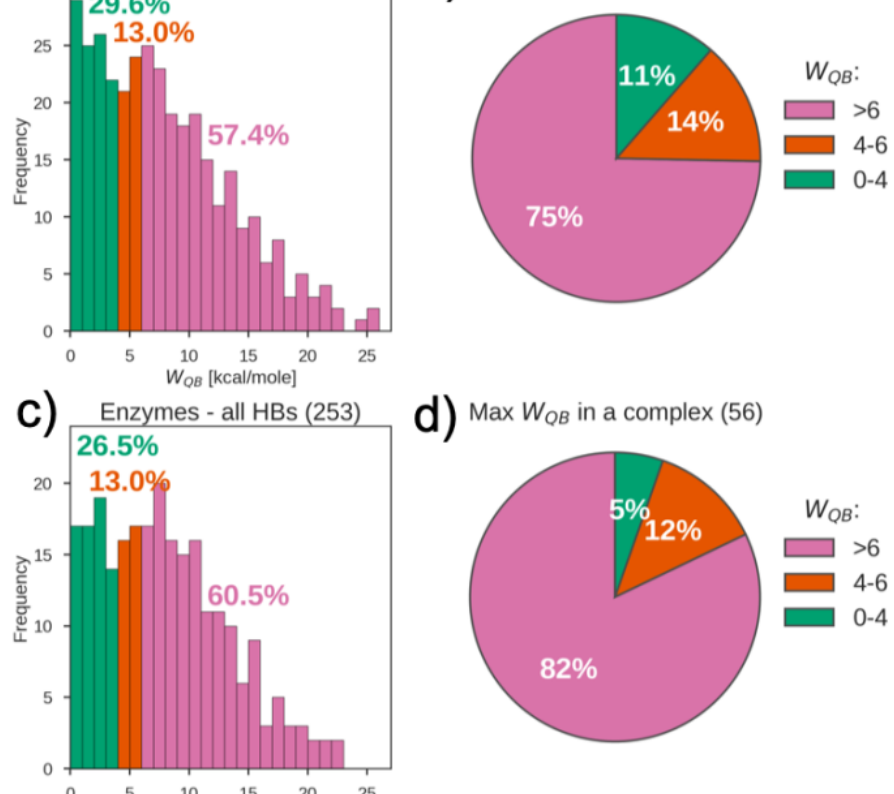

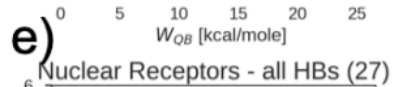
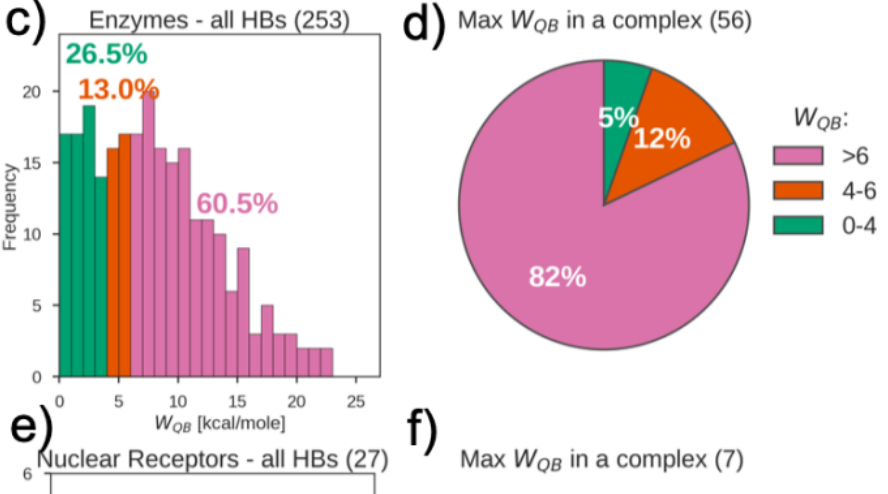

f)

$\operatorname{Max} W_{Q B}$ in a complex (7)
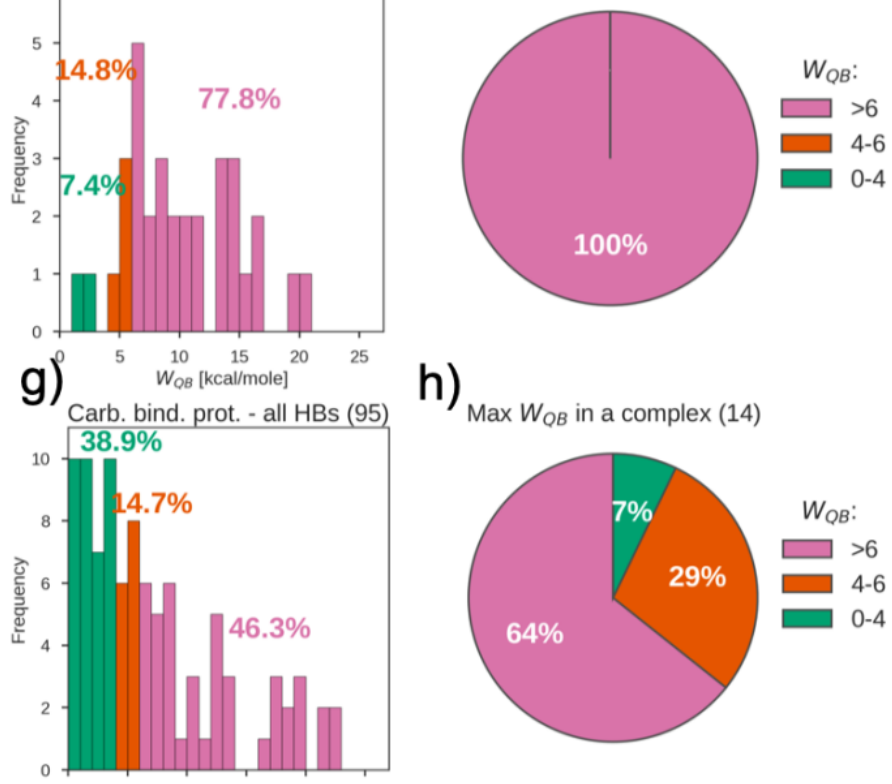

h) $\operatorname{Max} W_{Q B}$ in a complex (14)

i) ${ }^{0}{ }^{5} W_{O B}^{10}[\mathrm{kcal} / \mathrm{mole}]^{20}$
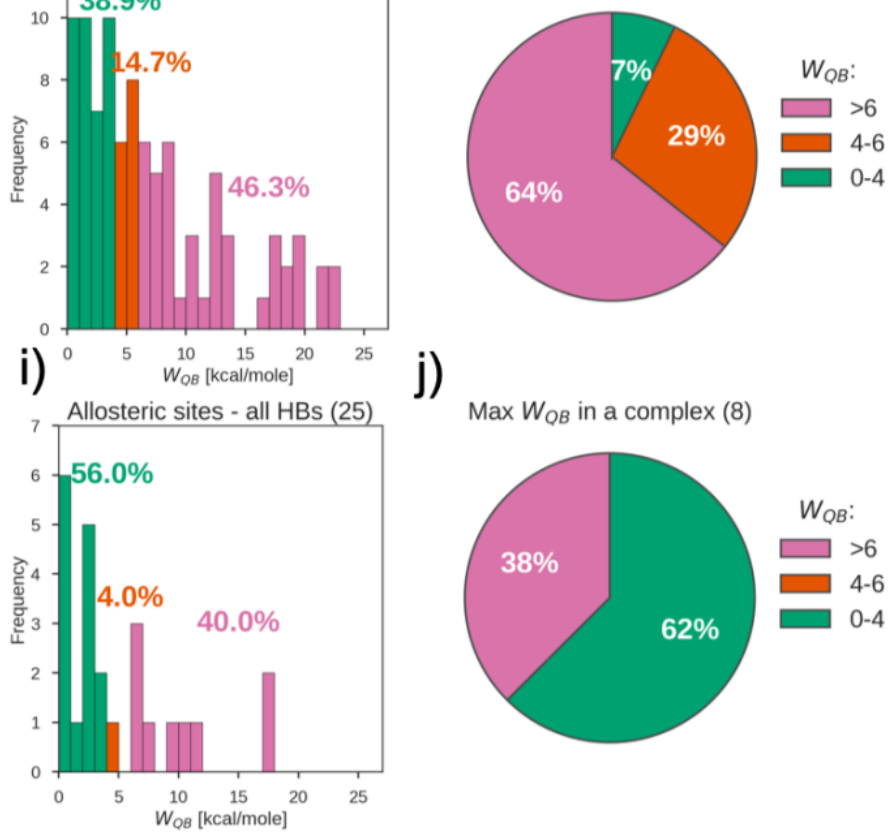

j)

Max $W_{Q B}$ in a complex (8)

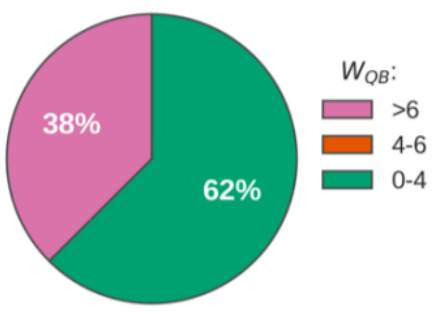


1 Figure 1 Histograms of frequency of $\mathrm{HBs}$ by $W_{Q B}$ value for: a) all simulated HBs (345), c) HBs

2 in enzyme active sites (253), e) HBs in the ligand binding site of nuclear receptors (27), g) HBs in 3 carbohydrate binding sites (95), i) HBs in allosteric sites (25). Pie charts showing share of 4 complexes with at least one robust $\mathrm{HB}\left(W_{Q B}>6 \mathrm{kcal} \mathrm{mol}^{-1}\right.$, pink), all labile $\mathrm{HBs}\left(W_{Q B}<4 \mathrm{kcal}\right.$ $5 \mathrm{~mol}^{-1}$, green) or intermediate situations (red) for: b) all simulated complexes (79), d) enzymes (56), 6 f) nuclear receptors (7), h) carbohydrate binding site (14), j) allosteric sites (8).
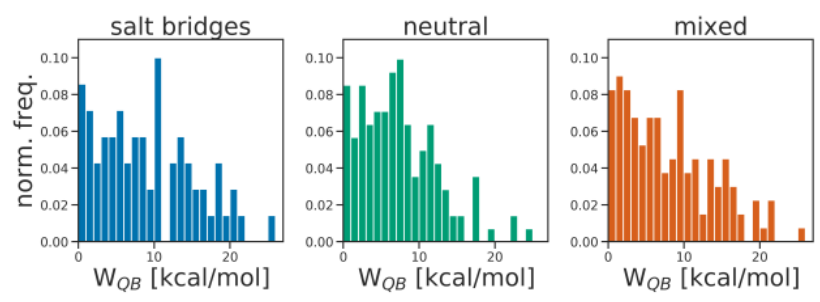

Figure 2 Histograms presenting distribution of $W_{Q B}$ values in the set of HBs from Iridium DS,

9 divided into salt bridges, neutral and mixed (ion-neutral) interactions.

11 strong indication that the behavior is dictated by the nature of the receptor. The proportion of 12 robust complexes increases to $82 \%$ in the case of enzyme active sites, which speaks about the need 13 of keeping the substrate in place for efficient catalysis. Nuclear receptors form fewer HBs with 14 their ligands, but most of them (78\%) are robust and all ligands (100\%) are well anchored. In this 15 case, forming a rigid structure may be necessary to stabilize the AF2 co-regulatory protein binding surface in an optimal conformation for co-activator binding. ${ }^{26}$ Carbohydrate binding sites, on the

17 other hand, form many more HBs with their ligands, but a lower proportion of robust ones (46\%).

18 Finally, in the case of allosteric ligands, only $40 \%$ of complexes are robust, suggesting that these 19 sites tend to yield looser complexes. As demonstrated in the case of HIV reverse transcriptase 20 inhibitors (Fig.3C), lack of robust HBs does not preclude tight binding. In fact, a multiplicity of 
1 binding modes might be beneficial to preserve binding affinity when the target is mutated, thus

2 averting resistance. ${ }^{27,28}$ While the distribution of HB strength between the four types of binding

3 sites that we have defined is quite different (see Supplementary Tables 4 and 5 for statistical tests),

4 individual cases can deviate from the norm (e.g. the allosteric ligand 1YV3 is extremely robust)

5 and more examples will be needed to reach firm conclusion about site-dependence.

6

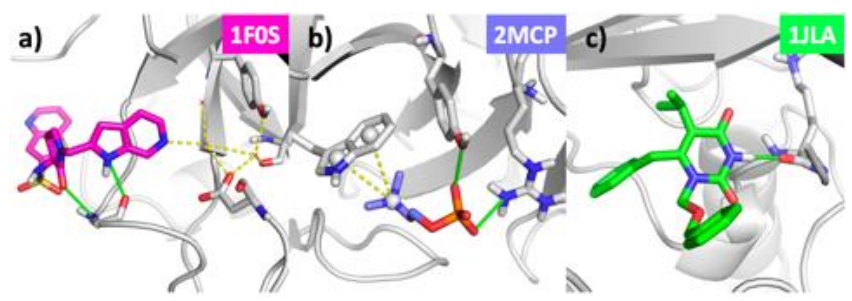

Figure 3 Structures of protein-ligand complexes that form potentially labile structures (all HBs weaker than $4 \mathrm{kcal} / \mathrm{mol})$. Weak hydrogen bonds ( $\left.\mathrm{W}_{\mathrm{QB}}<4 \mathrm{kcal} / \mathrm{mol}\right)$ marked in green. a) Complex of FXa with inhibitor RPR208707 (PDB id 1F0S; $K_{i}=18 \mathrm{nM}$ ) forms two direct, but labile, HBs with the protein. An additional water-mediated HB with the catalytic residues (yellow dotted lines) might provide structural stability. b) An antibody that recognizes phosphocholine (PDB id 2MCP) forms two charge-reinforced but labile HBs. A cation-pi interaction (yellow dotted lines) might provide structural stability. c) Reverse transcriptase inhibitor (PDB id $1 \mathrm{JLA} ; C_{50}=6 \mathrm{nM}$ ) forms a single but labile HB with the protein. No other source of structural stability is apparent.

We analyzed the distribution of robust HBs and found that they tend to concentrate on one part of the ligand (Supplementary Fig.4). To better understand this observation, all HBs in each complex were clustered, based on their distance in space, into fragment-sized group of atoms (Supplementary Fig.5). In the majority of complexes (62\%) robust HBs were located in a single group, forming a strong structural anchor (Fig.4, Supplementary Table 6). The concentration of robust interactions on a single site, allowing a some degree of movement to the other parts, minimises the entropic costs and can be desirable from a binding affinity perspective. ${ }^{6}$ Only $23 \%$ 
1 of ligands form two structural anchors on separate regions, though this is more common in the case

2 of carbohydrate-binding proteins (Supplementary Table 7). Three exceptional ligands manage to

3 form 3 distinct stable anchors. Interestingly, they have completely unrelated functions, chemical

4 structures and physical properties but - at least in two of those cases - there is a possible functional 5 explanation for the extreme robustness (Fig.5).
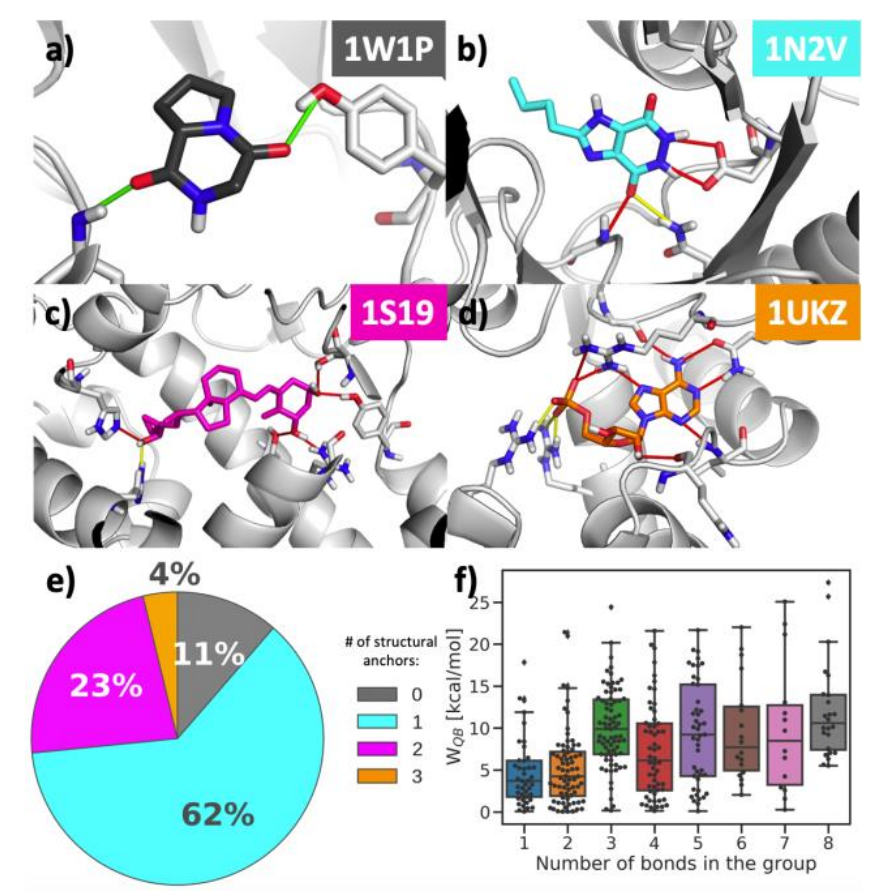

7 Figure 4 Division of complexes based on the number of structural anchors. Representative of each

8 group is presented in the following image: a) 0 anchors: Chitinase B with inhibitor (PDB id 1W1P;

$9 \quad \mathrm{IC}_{50}=5 \mathrm{mM}$ ); b) 1 anchor: Queuine tRNA-ribosyltransferase with inhibitor (PDB id 1N2V; $K_{i}=$

$1083 \mu \mathrm{M})$, c) 2 anchors: Vitamin D3 receptor with calcipotriol (PDB id $\left.1 \mathrm{~S} 19 ; K_{d}=0.31 \mathrm{nM}\right)$ and d)

113 anchors: Uridylate kinase - AMP (PDB id 1UKZ). e) Pie chart presenting distribution of number

12 of anchors across the data set. f) Distribution of strength of $\mathrm{HBs}_{\mathrm{Q}}\left(\mathrm{W}_{\mathrm{QB}}\right)$ versus the number of $\mathrm{HBs}$

13 per group of atoms. Weak hydrogen bonds $\left(\mathrm{W}_{\mathrm{QB}}<4 \mathrm{kcal} / \mathrm{mol}\right)$ marked in green, medium $\left(4 \leq \mathrm{W}_{\mathrm{QB}}\right.$

$14<6 \mathrm{kcal} / \mathrm{mol})$ in yellow and strong $\left(\mathrm{W}_{\mathrm{QB}} \geq 6 \mathrm{kcal} / \mathrm{mol}\right)$ in red. 


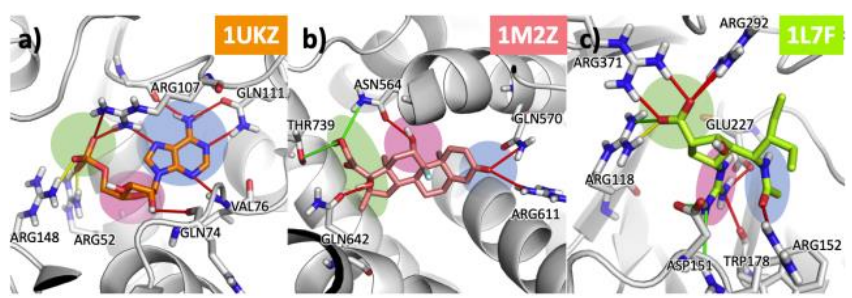

2 Figure 5 Structures of complexes with three binding anchors (shaded areas). a) Uridilate kinase with AMP (PDB id 1UKZ) where the base, ribose and phosphate of the nucleotide are forming

4 three distinctive centres of interactions. Structural stability may be necessary for efficient catalysis. b) Glucocorticoid receptor ligand-binding domain bound to dexamethasone (PDB id 1M2Z; $K_{d}=$ $19 \mathrm{nM})$. The ligand has three regions that form robust interaction, well separated in space but

7 located on the steroid core, thus behaving as a single rigid block. Structural stability may be necessary for agonistic response. c) Influenza virus neuraminidase with inhibitor BCX-1812 (PDB

9 id $1 \mathrm{~L} 7 \mathrm{~F} ; K_{i}$ single digit $\mathrm{nM}$ for various virus strains). Three different functional groups branching

10 out of the pentane scaffold form robust interactions in this extremely polar and solvent exposed

11 binding site. Weak hydrogen bonds $\left(\mathrm{W}_{\mathrm{QB}}<4 \mathrm{kcal} / \mathrm{mol}\right)$ marked in green, medium $\left(4 \leq \mathrm{W}_{\mathrm{QB}}<6\right.$

$12 \mathrm{kcal} / \mathrm{mol})$ in yellow and strong $\left(\mathrm{W}_{\mathrm{QB}} \geq 6 \mathrm{kcal} / \mathrm{mol}\right)$ in red.

13 The distribution of $W_{Q B}$ per number of HBs in a local group (Fig. $4 \mathrm{f}$ ) is suggestive of cooperative

14 behavior. HBs in isolation usually do not form robust interactions (mean and median values: (4.7

$15 \pm 4.1$ ) and $3.7 \mathrm{kcal} \mathrm{mol}^{-1}$, respectively), although in exceptional cases they can reach values above

$1610 \mathrm{kcal} \mathrm{mol}^{-1}$. By contrast, when three or more HBs cluster together, formation of robust complexes

17 is the most common outcome (mean and median values: $(9.4 \pm 5.8)$ and $9.0 \mathrm{kcal} \mathrm{mol}^{-1}$, 18 respectively). The HBs within these clusters present relatively similar $\mathrm{W}_{\mathrm{QB}}$ values (Supplementary 19 Fig.6), suggesting that they often behave in a concerted-like manner. This synergic and mutually dependent behavior not only ensures higher barriers to dissociation, but is also well-suited to 
1 provide selectivity, as small changes in the composition or geometry of one of the partners may

2 result in large changes in magnitude of $\mathrm{W}_{\mathrm{QB}}$ (see example in Supplementary Fig.7).

3 The observation that most drug-like ligands combine tightly-bound regions with looser makes

4 us wonder about fragment-sized ligands. Do they valance order and disorder in some other way

5 (e.g. using fewer attachment points)? Or, perhaps, depending on the site they bind to, they are

6 either dynamic or fully constrained? In order to answer these questions, we have extended our

7 analysis with a set of 27 fragment-protein complexes (126 individual HBs) from the SERAPhiC

8 dataset. ${ }^{29}$ Strikingly, we find that fragments have an almost identical behavior to standard ligands,

9 with $49 \%$ of robust HBs (2.3 per ligand) and $73 \%$ of ligands presenting at least one robust

10 interaction. The distribution and maximal $\mathrm{W}_{\mathrm{QB}}$ values are also very similar (Fig.6). This indicates

11 that, proportionally, fragments are more static than standard ligands. This agrees with the

12 observations that fragments have a more enthalpic binding ${ }^{30}$ and that they have a higher proportion

13 of buried HBs. ${ }^{31}$ It also justifies that, in spite of their low binding affinity, most fragments already

14 have a well-defined binding mode that serves as a foundation from which to spread and catch

15 additional interactions. However, not all fragments form robust interactions and we propose that

16 these are less suitable as starting points because their binding mode can change, confounding

17 structure-activity interpretation and rendering optimization more difficult. Indeed, fragments are

18 known to change their binding mode when evolved into larger molecules. ${ }^{7,32-36}$ These may be

19 attempts at building on what is assumed to be a solid foundation but turns out to be unstable

20 ground, a possibility that we shall investigate in the future. It should also be noted that the fraction

21 of well-anchored fragments may be different for fragments hits that fail to crystallize. The overlap

22 between X-ray crystallography and other biophysical screening methods can be rather low ${ }^{37}$ and

23 progressing fragments that fail to crystallize is deemed difficult but worthwhile. ${ }^{38}$ 
a)

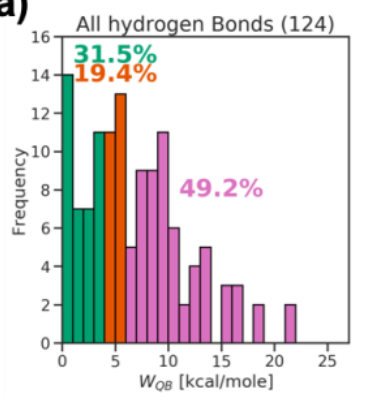

b)

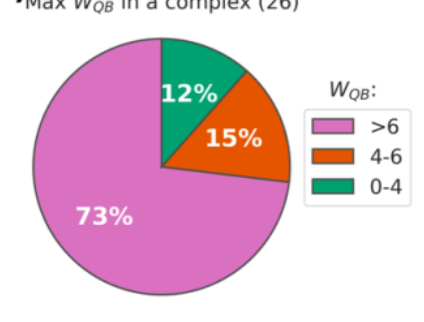

Figure 6 a) Histograms of frequency of HBs by $W_{Q B}$ value for all simulated HBs (124) in SERAPhiC dataset. Pie charts showing share of complexes with at least one robust $\mathrm{HB}\left(W_{Q B}>6\right.$ $\mathrm{kcal} \mathrm{mol}^{-1}$, pink), all labile $\mathrm{HBs}\left(W_{Q B}<4 \mathrm{kcal} \mathrm{mol}^{-1}\right.$, green) or intermediate situations (red) for all protein-fragment complexes (26).

Finally, we want to consider what is the origin of the free energy barrier that causes structural stability. Knowing that a HB has a large $W_{Q B}$ value can be likened to knowing the $k_{\text {off }}$ of a compound without knowing the $k_{\text {on }}$ nor $\Delta G_{b i n d}$ : larger values may indicate that it has a higher transition state (if $\Delta G_{\text {bind }}$ remains the same; Fig.7a), that the complex is thermodynamically more stable (if $k_{\text {on }}$ remains the same; Fig. $7 \mathrm{~b}$ ), or a combination thereof. In this data set, we find that anchoring sites often correspond to binding hot spots. This is indeed the case for all kinases and proteases, which have a well-known binding hot spot (Supplementary Table 3, Supplementary Fig.5), as well as for most fragments. In such cases, $\Delta G_{b i n d}$ must be a component of $W_{Q B}$, but there is no correlation between both magnitudes (Supplementary Fig.8), as already noted. ${ }^{20}$ Thus, we conclude that $W_{Q B}$ must be largely dominated by a transitory dissociation penalty. The origin of this penalty can be explained by a physical decoupling between HB rupture and resolvation, as described for water-shielded hydrogen bonds. ${ }^{19}$ In support of this view, several studies of the reverse event have identified desolvation of the binding pocket as the rate-limiting step in ligand association. ${ }^{18,39,40}$ Indeed, solvent exposed HBs invariably lead to low $W_{Q B}$ values (but note that 
1 they can be thermodynamically stable), ${ }^{41}$ whereas water-shielding is a necessary but not sufficient

2 condition of robust HBs (Supplementary Fig.9).
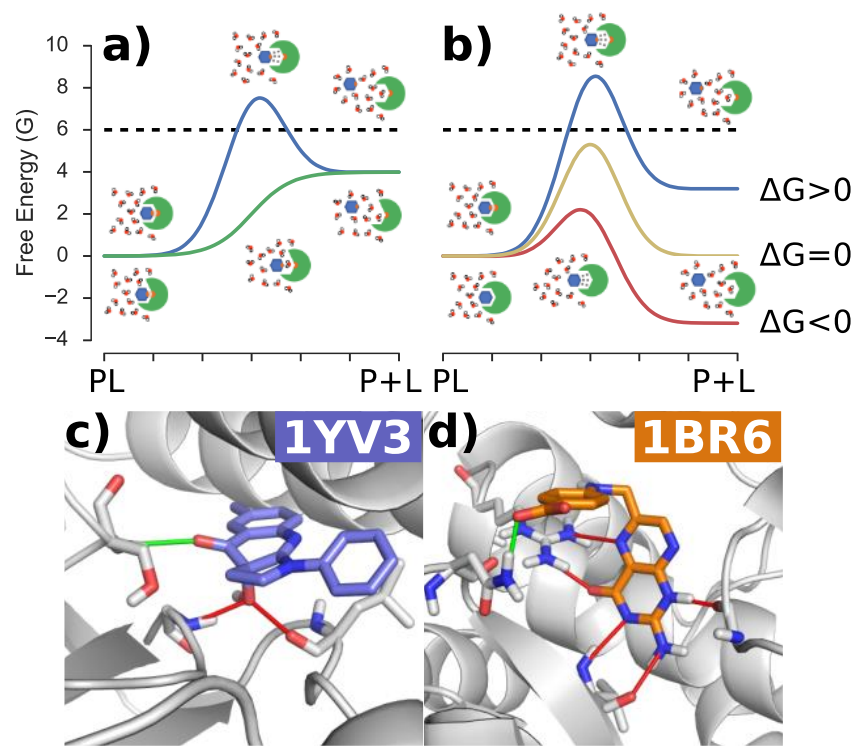

4 Figure 7 Ways of achieving structural robustness. a) Idealized representation of two dissociation

5 pathways for complexes with the same $\Delta G_{b i n d}$ and different desolvation costs. The images above

6 the blue curve shows the state of the system in bound, transition and unbound state of complex

7 with well shielded, stable hydrogen bond. The images below the green curve show analogous

8 images for the complex with solvent exposed hydrogen bond. b) Likewise for two complexes with

9 the same desolvation cost but different $\Delta G_{b i n d}$. The images represent complexes with excellent

10 shape complementarity that form (above the blue curve) or don't form (below the red curve)

11 favorable hydrogen-bonding pairs. The black dashed line marks the energy cutoff that classifies

12 bond as structurally stable. c) Example of a complex with high dissociation cost due to extreme

13 water-shielding. d) Example of a complex with high dissociation cost due to a tight network of

14 multiple HBs. Weak hydrogen bonds ( $\left.\mathrm{W}_{\mathrm{QB}}<4 \mathrm{kcal} / \mathrm{mol}\right)$ marked in green and strong $\left(\mathrm{W}_{\mathrm{QB}} \geq 6\right.$

$15 \mathrm{kcal} / \mathrm{mol})$ in red. 
Taken together, our results show that structural stability is a common property of protein-ligand complexes, but not an universal one. Cases of loose complexes, while relatively rare (10-20\%),

4 can be found even in a dataset originating exclusively from X-ray crystallography, a technique that 5 requieres structural homogeneity of the sample. The proportion could be larger amongst ligands

6 that fail to crystallize. The level of residual mobility is also larger and more common than the static 7 X-ray structures lead to think, as also concluded by a recent independent study. ${ }^{4}$ In fact, most 8 complexes balance order and disorder by combining a firm anchor with more relaxed peripheral 9 interactions. Depending on the nature of the ligand and the binding site, each complex adopts a 10 particular degree of robustness, that ranges from the very tight (e.g. nuclear receptor agonists) to 11 the very loose (e.g. HIV-RT allosteric inhibitors). Each one of these solutions entails important 12 consequences that have, so far, been neglected in drug design. First of all, a firm anchor provides 13 a framework from which to grow and capture additional interactions, and the preservation of a 14 common binding mode helps interpreting structure-activity relationships. This is particularly 15 important for fragments as starting points for lead discovery. Secondly, structural robustness can 16 have functional implications, particularly in the case of receptors, where flexibility has been linked 17 to the agonist/antagonist response..$^{26,42}$ Thirdly, structural stability implies an entropic penalty and 18 must be balanced to avoid loss of potency. ${ }^{6,43}$ Finally, the deep and narrow energetic minima that 19 cause rigidity also imply large penalties for small recognition defects, thus increasing the fidelity 20 of the recognition event. This has been shown for protease-substrate pairs ${ }^{44}$ and HIV-protease 21 inhibitors. ${ }^{45}$ In conclusion, this work opens up the possibility of understanding and designing 22 structural robustness in ligand-receptor complexes. We suggest that robustness analysis, which can 23 help understand and control the level of mobility, should be an essential part of ligand design, not 
1 least because rigid parts demand more precise complementarity than flexible ones. Qualitatively,

2 a visual inspection can reveal water-shielded HBs (Fig.7c) and HB clusters (Fig.7d), which are

3 tell-tale signs of robustness. Quantitatively, DUck simulations offer an inexpensive and automated

4 protocol to calculate $W_{Q B}$. While HBs appear to be the most common means of achieving structural

5 robustness, other interaction types (e.g. cation-pi, water-mediated HBs, halogen bonds) should be

6 considered in the future.

9 Supporting Information. Detailed methods, additional figures and tables. Set of sdf files

10 containing ligands and mol2 files containing protein structures.

\section{AUTHOR INFORMATION}

\section{Corresponding Author}

13 *E-mail: xbarril@ub.edu

\section{ACKNOWLEDGMENT}

15 We thank C. Galdeano, F. J. Luque and the anonymous reviewers for helpful discussions and 16 manuscript revision. The research was funded under the EU Horizon 2020 programme, Marie

17 Skłodowska-Curie grant agreement No. 675899 (FRAGNET). Spanish Ministerio de Economia 18 (SAF2015-68749-R). Catalan government (2014 SGR 1189). Barcelona Supercomputing Center 19 (computational resources). 
1 X.B. designed the project; M.M. performed calculations; S.R.C. contributed new analytic tools;

2 M.M. and X.B. analysed data and wrote the manuscript.

\section{REFERENCES}

4 1. Tompa, P. \& Fuxreiter, M. Fuzzy complexes: polymorphism and structural disorder in

5 protein-protein interactions. Trends Biochem. Sci. 33, 2-8 (2008).

6 2. Borgia, A. et al. Extreme disorder in an ultrahigh-affinity protein complex. Nature 555, 61$766(2018)$

3. Cozzini, P. et al. Target Flexibility: An Emerging Considertaion in Drug Discovery. J. Med. Chem. 51, 6237-6255 (2008).

10 4. Van Zundert, G. C. P. et al. QFit-ligand Reveals Widespread Conformational Heterogeneity 11 of Drug-Like Molecules in X-Ray Electron Density Maps. J. Med. Chem. 61, 11183-11198 (2018).

13 5. Klebe, G. Applying thermodynamic profiling in lead finding and optimization. Nature Reviews Drug Discovery 14, 95-110 (2015).

6. Glas, A., Wamhoff, E.-C., Kruger, D. M., Rademacher, C. \& Grossmann, T. N. Increased its binding mode? J. Med. Chem. 60, 128-145 (2017). of aspartic protease inhibitors. Angew. Chemie - Int. Ed. 54, 2849-2853 (2015). 
$19 . \quad$ Sliwoski, G., Kothiwale, S., Meiler, J. \& Lowe, E. W. Computational methods in drug

2 discovery. Pharmacol. Rev. 66, 334-95 (2014).

3 10. Pan, A. C., Borhani, D. W., Dror, R. O. \& Shaw, D. E. Molecular determinants of drug 4 receptor binding kinetics. Drug Discov. Today 18, 667 (2013).

5 11. Bissantz, C., Kuhn, B. \& Stahl, M. A Medicinal Chemist's Guide to Molecular Interactions.

$6 \quad$ J. Med. Chem. 53, 5061-5084 (2010).

7 12. Ferreira de Freitas, R. \& Schapira, M. A systematic analysis of atomic protein-ligand 8 interactions in the PDB. Med. Chem. Commun. 8, 1970-1981 (2017).

9 13. Fersht, A. R. The hydrogen bond in molecular recognition. Trends Biochem. Sci. 12, 301$10304(1987)$.

11 14. Pace, C. N. Energetics of protein hydrogen bonds. Nat. Struct. Mol. Biol. 16, 681-682 12 (2009).

13 15. Pace, C. N. et al. Contribution of hydrogen bonds to protein stability. Protein Sci. 23, 652$14 \quad 661(2014)$.

15 16. Gao, J., Bosco, D. A., Powers, E. T. \& Kelly, J. W. Localized thermodynamic coupling 16 between hydrogen bonding and microenvironment polarity substantially stabilizes proteins. 17 Nat. Struct. Mol. Biol. 16, 684-690 (2009).

18 17. Nick Pace, C., Martin Scholtz, J. \& Grimsley, G. R. Forces stabilizing proteins. FEBS Lett. $19 \quad \mathbf{5 8 8}, 2177-2184(2014)$.

20 18. Mondal, J., Friesner, R. A. \& Berne, B. J. Role of Desolvation in Thermodynamics and 
Kinetics of Ligand Binding to a Kinase. J. Chem. Theory Comput. 10, 5696-5705 (2014).

2

19. Schmidtke, P., Javier Luque, F., Murray, J. B. \& Barril, X. Shielded hydrogen bonds as structural determinants of binding kinetics: Application in drug design. J. Am. Chem. Soc. 133, 18903-18910 (2011).

20. Ruiz-carmona, S. et al. Dynamic undocking and the quasi-bound state as tools for drug discovery. Nat. Chem. 9, 201 (2017).

21. Warren, G. L., Do, T. D., Kelley, B. P., Nicholls, A. \& Warren, S. D. Essential considerations for using protein-ligand structures in drug discovery. Drug Discov. Today 17, 1270-1281 (2012).

22. Majewski, M., Ruiz-Carmona, S. \& Barril, X. in Rational Drug Design: Methods and Protocols (eds. Mavromoustakos, T. \& Kellici, T. F.) 195-215 (Springer New York, 2018). doi:10.1007/978-1-4939-8630-9_11

23. Kleywegt, G. J. \& Jones, T. A. in Methods in enzymology 208-230 (Elsevier, 1997).

24. Parthasarathy, S. \& Murthy, M. R. N. On the correlation between the main-chain and sidechain atomic displacement parameters (B values) in high-resolution protein structures. Acta Crystallogr. Sect. D Biol. Crystallogr. 55, 173-180 (1999).

25. Barril, X., Aleman, C., Orozco, M. \& Luque, F. J. Salt Bridge Interactions : Stability of the Ionic and Neutral Complexes in the Gas Phase, in Solution, and in Proteins. Proteins Struct. Funct. Bioinforma. 32, 67-79 (1998).

26. Mayer-Wrangowski, S. C. \& Rauh, D. Monitoring ligand-induced conformational changes for the identification of estrogen receptor agonists and antagonists. Angew. Chemie - Int. 
Ed. 54, 4379-4382 (2015).

2 27. Das, K. et al. Roles of Conformational and Positional Adaptability in Structure-Based 3 Design of TMC125-R165335 ( Etravirine) and Related Non-nucleoside Reverse $4 \quad$ Transcriptase Inhibitors That Are Highly Potent and Effective against Wild-Type and Drug5 Resistant HIV-1 Vari. J. Med. Chem. 47, 2550-2560 (2004).

6 28. Lee, W. G., Chan, A. H., Spasov, K. A., Anderson, K. S. \& Jorgensen, W. L. Design, Conformation, and Crystallography of 2-Naphthyl Phenyl Ethers as Potent Anti-HIV

9 29. Favia, A. D., Bottegoni, G., Nobeli, I., Bisignano, P. \& Cavalli, A. SERAPhiC : A Benchmark for in Silico Fragment-Based Drug Design. J. Chem. Inf. Model. 51, 2882-2896 (2011).

12 30. Ferenczy, G. G. \& Keseru, G. M. Thermodynamics of fragment binding. J. Chem. Inf. $13 \quad$ Model. 52, 1039-1045 (2012).

14 31. Giordanetto, F., Jin, C., Willmore, L., Feher, M. \& Shaw, D. E. Fragment hits: What do they look like and how do they bind? J. Med. Chem. 62, 3381-3394 (2019).

16 32. Drwal, M. N. et al. Structural insights on fragment binding mode conservation. J. Med. 17 Chem. (2018). doi:10.1021/acs.jmedchem.8b00256

18 33. Mpamhanga, C. P. et al. One scaffold, three binding modes: Novel and selective pteridine reductase 1 inhibitors derived from fragment hits discovered by virtual screening. $J$. Med. Chem. 52, 4454-4465 (2009).

21 34. Casale, E. et al. Fragment-based hit discovery and structure-based optimization of 
aminotriazoloquinazolines as novel Hsp90 inhibitors. Bioorganic Med. Chem. 22, 41354150 (2014).

35. Han, X. et al. Discovery of potent and selective CDK8 inhibitors through FBDD approach. Bioorganic Med. Chem. Lett. 27, 4488-4492 (2017).

36. Forster, A. B. et al. The identification of a novel lead class for phosphodiesterase 2 inhibition by fragment-based drug design. Bioorganic Med. Chem. Lett. 27, 5167-5171 (2017).

37. Schiebel, J. et al. Six Biophysical Screening Methods Miss a Large Proportion of Crystallographically Discovered Fragment Hits: A Case Study. ACS Chem. Biol. 11, 16931701 (2016).

38. Erlanson, D. A., Davis, B. J., Jahnke, W. \& Box, G. Perspective Fragment-Based Drug Discovery: Advancing Fragments in the Absence of Crystal Structures. Cell Chem. Biol. 1-7 (2019). doi:10.1016/j.chembiol.2018.10.001

39. Dror, R. O. et al. Pathway and mechanism of drug binding to G-protein-coupled receptors. Proc. Natl. Acad. Sci. U. S. A. 108, 13118-23 (2011).

40. Schuetz, D. A. et al. Ligand Desolvation steers on-rate and impacts Drug Residence Time of Heat shock protein 90 (Hsp90) Inhibitors. J. Med. Chem. 90, (2018).

41. Ciulli, A., Williams, G., Smith, A. G., Blundell, T. L. \& Abell, C. Probing hot spots at protein-ligand binding sites: A fragment-based approach using biophysical methods. $J$. Med. Chem. 49, 4992-5000 (2006).

42. Ghanouni, P. et al. Functionally Different Agonists Induce Distinct Conformations in the G 
Protein Coupling Domain of the $\beta 2$ Adrenergic Receptor. J. Biol. Chem. 276, 24433-24436 (2001).

3 43. Brandt, T. et al. Congeneric but still distinct: How closely related trypsin ligands exhibit different thermodynamic and structural properties. J. Mol. Biol. 405, 1170-1187 (2011).

44. Fuchs, J. E. et al. Cleavage Entropy as Quantitative Measure of Protease Specificity. PLoS Comput. Biol. 9, e1003007 (2013).

45. Shen, Y., Radhakrishnan, M. L. \& Tidor, B. Molecular mechanisms and design principles inhibition. Proteins Struct. Funct. Bioinforma. 83, 351-372 (2015). 